\title{
Peanut Sprout Extracts Cultivated with Fermented Sawdust Medium Inhibits Benign Prostatic Hyperplasia In Vitro and In Vivo
}

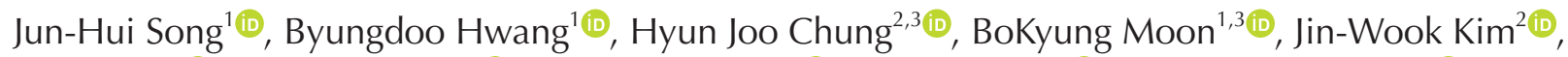

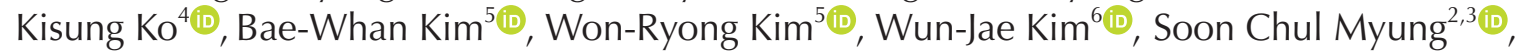 \\ Sung-Kwon Moon ${ }^{1,3}$ (iD \\ ${ }^{1}$ Department of Food and Nutrition, College of Biotechnology and Natural Resources, Chung-Ang University, Anseong, ${ }^{2}$ Department of \\ Urology, Chung-Ang University College of Medicine, ${ }^{3}$ Bio-Integration Research Center for Nutra-Pharmaceutical Epigenetics, Chung- \\ Ang University, ${ }^{4}$ Department of Medicine, Chung-Ang University College of Medicine, Seoul, ${ }^{5}$ Resvera Co., Ltd., ${ }^{6}$ Department of Urology, \\ Chungbuk National University College of Medicine, Cheongju, Korea
}

Purpose: In this study, we tested whether the resveratrol-enriched peanut sprout extracts cultivated with fermented sawdust medium (PSEFS) could suppress benign prostatic hyperplasia (BPH) in vitro and in vivo.

Materials and Methods: The mode of action of PSEFS was estimated by employing high-performance liquid chromatography analysis, MTT assay, cell counting, cell cycle analysis, immunoblots, and immunoprecipitation and electrophoretic mobility shift assay. In vivo efficacy of PSEFS was analyzed in BPH animal model via immunostaining and enzyme-linked immunosorbent assay.

Results: We selected the Yesan peanut sprout variety, which contains the highest level of resveratrol. The resveratrol levels in PSEFS were higher than those obtained with hydroponic technology. PSEFS treatment induced cell cycle arrest at the G1phase by downregulating CDK4 and cyclin D1 via p21WAF1 induction in the RWPE-1 and WPMY prostate cells, thereby decreasing their proliferation. Treatment with PSEFS decreased ERK1/2 phosphorylation and increased JNK phosphorylation. The levels of DNA-bound transcription factors associated with proliferation (nuclear factor- $\mathrm{B}$, Sp-1, and AP-1) decreased upon PSEFS treatment in both prostate cells. Additionally, the levels of the molecular markers of BPH development $(5 \alpha$-reductase, androgen receptor, fibroblast growth factor, $\mathrm{BCl}-2$, and Bax) also changed by the addition of PSEFS. Finally, in a testosterone propionate-induced $\mathrm{BPH}$ model in rats, PSEFS administration attenuated the size, weight, and thickness of prostate tissues with no signs of death.

Conclusions: These results showed that PSEFS inhibited BPH both in vitro and in vivo and might be useful in the development of a potential BPH therapy.

Keywords: Benign prostatic hyperplasia; Cell cycle; Peanut; Resveratrol; RWPE-1; Sawdust

This is an Open Access article distributed under the terms of the Creative Commons Attribution Non-Commercial License (http://creativecommons.org/licenses/by-nc/4.0) which permits unrestricted non-commercial use, distribution, and reproduction in any medium, provided the original work is properly cited.

Received: Jan 3, 2020 Revised: Feb 13, 2020 Accepted: Feb 15, 2020 Published online Mar 19, 2020

Correspondence to: Sung-Kwon Moon (iD https://orcid.org/0000-0002-0260-7231

Department of Food and Nutrition, College of Biotechnology and Natural Resources, Chung-Ang University, 4726 Seodong-daero, Daedeokmyeon, Anseong 17546, Korea.

Tel: +82-31-670-3284, Fax: +82-31-675-4853, E-mail: sumoon66@cau.ac.kr

Correspondence to: Soon Chul Myung (iD https://orcid.org/0000-0002-6727-5450

Department of Urology, Chung-Ang University College of Medicine, 84 Heukseok-ro, Dongjak-gu, Seoul 06974, Korea.

Tel: +82-2-6299-1808, Fax: +82-2-813-5387, E-mail: uromyung@cau.ac.kr 


\section{INTRODUCTION}

Benign prostatic hyperplasia $(\mathrm{BPH})$ is one of the most common urological diseases in men over 50 years old and is characterized by nonmalignant enlargement of the prostate gland [1]. In $\mathrm{BPH}$, the proliferation of both epithelial and stromal cells within the transitional zone of the prostate leads to the development of lower urinary tract symptoms, including urinary hesitancy, frequent urination, weak stream, and nocturia [2]. In addition, BPH development is associated with increased levels of dihydrotestosterone (DHT), produced from testosterone by the $5 \alpha$-reductase enzyme within the prostate glands [3]. Thus, management of the key molecular events associated with the uncontrolled proliferation of prostate cells and the activity of the $5 \alpha$-reductase enzyme is a critical target for the development of preventive and therapeutic agents. The pharmacological inhibition of $5 \alpha$-reductase by finasteride or dutasteride has been used in the treatment of BPH [4]. However, these drugs have adverse side effects [5,6]. Therefore, novel therapeutic agents that are natural, tolerable, and efficient are necessary for the treatment of BPH.

Many factors, such as hormones and growth factors, play important roles in the pathogenesis of $\mathrm{BPH}$. Binding of DHT to the androgen receptor (AR) induces conformational changes that lead to abnormal growth of the prostate through subsequent molecular events $[7,8]$. During the progression of $\mathrm{BPH}$, prostatic cells including epithelial cells and stromal cells proliferate via critical steps involving dysregulated cell-cycle progression, altered signaling pathways, and enhanced transcription factors expression [9-11]. Therefore, such molecular changes that occur during $\mathrm{BPH}$ progression are other potential targets for developing preventive or therapeutic agents.

Resveratrol (trans-3,5,4'-trihydroxy stilbene, RES) is a polyphenolic compound that is found in plants as a natural phytoalexin [12]. It has been reported that RES acts as an effective natural antioxidant by scavenging free radicals and prevents malignant transformation [13]. In addition to grape and polygonum cuspidatum, peanut sprouts have been found as an economical dietary source of RES [14]. Kang et al [15] reported that peanut sprouts contain RES, which can be a source of natural antioxidants. Several studies in BPH have suggested that RES inhibits the proliferation of prostatic cells [16].
Hydroponic technology (HP) has been widely used to cultivate peanut sprouts without the use of soil. Although it is a cost-effective technique [17], it causes pollution due to the discharge of waste solutions [18]. Therefore, an environmentally sustainable technology with a higher output is needed. Sawdust medium is a promising option for the soilless cultivation of sprouts that have high nutritional quality [19]. During the fermentation of sawdust, the pathogens in the raw materials are killed while the nutrients beneficial to plant growth are enriched [20].

RES-enriched peanut sprout extracts have been shown to have various bioactivities, including antiinflammatory, anti-oxidative, and anti-obesity effects [15,21-23]. However, the physiological effects of the peanut sprout extracts cultivated with fermented sawdust medium (PSEFS) have not been investigated yet. In this study, we examined the RES levels in the PSEFS and compared them with that obtained by the conventional HP. In addition, the molecular effects of PSEFS on $\mathrm{BPH}$ were investigated in vitro and in vivo.

\section{MATERIALS AND METHODS}

\section{Materials}

RES ( $\geq 98 \%$, analytical standard grade) and highperformance liquid chromatography (HPLC)-grade solvents, including water, ethanol, and acetonitrile, were purchased from Burdick \& Jackson (Phillipsburg, NJ, USA). The other materials used are described under the Supplementary Materials and Methods section.

\section{Sample preparation}

The sawdust medium was kindly provided by Resvera Co. (Cheongju, Korea). Sample was obtained as described in the Supplementary Materials and Methods section.

\section{Resveratrol analysis}

For RES extraction, each sample was mixed well with methanol. The extracts were centrifuged, and the supernatant was filtered through a $0.45-\mu \mathrm{m}$ polytetrafluoroethylene syringe filter for the analysis. RES was analyzed using an HPLC system. The detailed method used are described under the Supplementary Materials and Methods section. 


\section{Cell culture, MTT assay, cell counting, cell cycle analysis, immunoblots, immunoprecipitation, and electrophoretic mobility shift assay}

The human normal prostate epithelial cells (RWPE1) and stromal cells (WPMY) were obtained from American Type Culture Collection. Cell culture, MTT assay, cell counting, cell cycle analysis, immunoblots, immunoprecipitation (IP), and electrophoretic mobility shift assay (EMSA) were performed as reported in the Supplementary Materials and Methods section.

\section{Ethics statement}

All animal experiments were reviewed and approved by the ethics committee for the care and use of laboratory animals at Chung-Ang University. (approval Number: 201900030). Animals, BPH Animal Model, and Immunohistochemistry were performed as reported in the Supplementary Materials and Methods section.

\section{Enzyme-linked immunosorbent assay}

To measure DHT levels, an ELISA kit specific to DHT (MyBioSource, San Diego, CA, USA) was used according to the manufacturer's instructions. The detailed method used are described under the Supplementary Materials and Methods section.

\section{Statistical analysis}

Statistical analyses were performed using IBM SPSS Statistics ver. 23.0.1 sof tware (IBM Corp., Armonk, NY, USA). Data are presented as mean \pm standard deviation (SD). Factorial analysis of variance (ANOVA) with post hoc Fisher's least significant difference test was utilized to analyze the data. $p<0.05$ was considered to indicate a statistically significant difference.

\section{RESULTS}

\section{The peanut sprouts cultivated with} fermented sawdust medium had higher amounts of resveratrol than those grown by hydroponic technology

The RES contents of four varieties of peanut sprouts germinated for 9 days using the sawdust medium fermented for 45 days were determined (Fig. 1A). The highest RES content $(16.74 \pm 1.49 \mu \mathrm{g} / \mathrm{g})$ was detected in Yesan, followed by Inhua-C $(9.23 \pm 0.28 \mu \mathrm{g} / \mathrm{g})$, Inhua-L $(7.79 \pm 0.83 \mu \mathrm{g} / \mathrm{g})$, Inhua-W $(6.12 \pm 0.50 \mu \mathrm{g} / \mathrm{g})$, and Sindae $(5.00 \pm 0.62 \mu \mathrm{g} / \mathrm{g})$. Therefore, the Yesan variety was selected for subsequent experiments. The RES contents of Yesan which was germinated using fermented sawdust medium were compared using the commercial peanut sprouts cultivated with the HP as a control. As shown in Fig. 1B, the RES content was significantly higher in Yesan than the commercial sample. Limmongkon et al [22] have also reported that RES contents ranged from 0.3 to $6.4 \mu \mathrm{g} / \mathrm{g}$ in peanut sprouts germinated by the HP method. These results were in accordance with our result regarding HP control $(4.14 \pm 0.56 \mu \mathrm{g} / \mathrm{g})$. The RES content of the PSEFS using Yesan variety was $24.56 \pm$ $0.54 \mu \mathrm{g} / \mathrm{g}$ (data not shown).

\section{Peanut sprout extracts cultivated with fermented sawdust medium inhibited proliferation of human normal prostate cells}

The human normal prostate epithelial cells (RWPE1) and stromal cells (WPMY) were used to investigate the effects of PSEFS. PSEFS treatment significantly suppressed the cell viability in RWPE-1 cells (Fig. 2B). Trypan Blue staining showed that PSEFS-treatment of

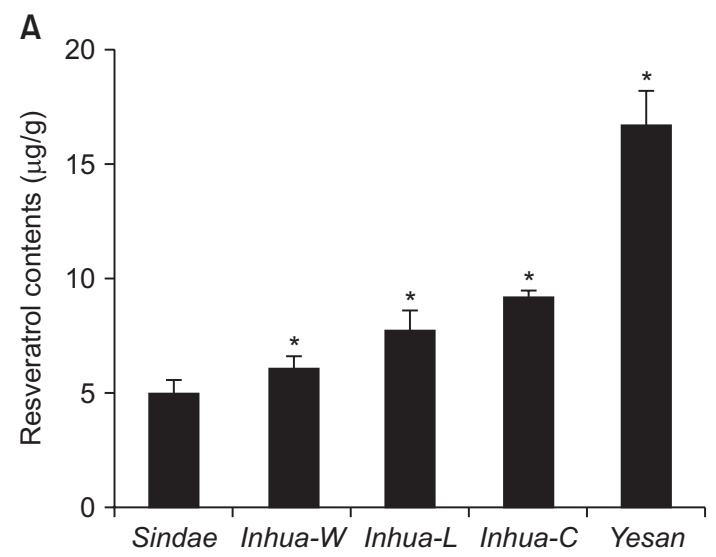

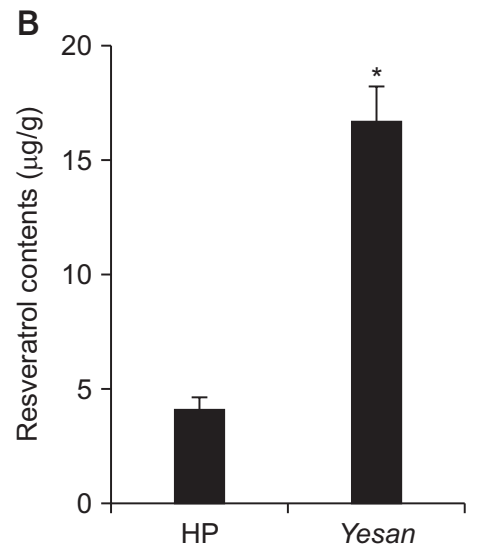

Fig. 1. (A) Resveratrol contents in differ-
ent varieties of peanut sprouts (Sindae,
Inhua-W, Inhua- $L$, Inhua-C, Yesan)
cultivated with sawdust medium fer-
mented for 45 days. (B) Resveratrol con-
tents in peanut sprouts cultivated with
hydroponics (HP) or sawdust medium
fermented for 45 days. Values in the
bar graph show mean \pm standard devia-
tion of three independent experiments.
*p<0.05, compared with the Sindae or
HP group. 
RWPE-1 cells reduced the number of viable cells (Fig. 2A). Similar results were observed in WPMY cells using both cell viability and counting assays (Fig. 2A, 2B). These results showed that PSEFS treatment inhibited the proliferation of human normal prostate cells, and the $\mathrm{IC}_{50}$ value was approximately $800 \mu \mathrm{g} / \mathrm{mL}$ in both RWPE-1 and WPMY cells.

A
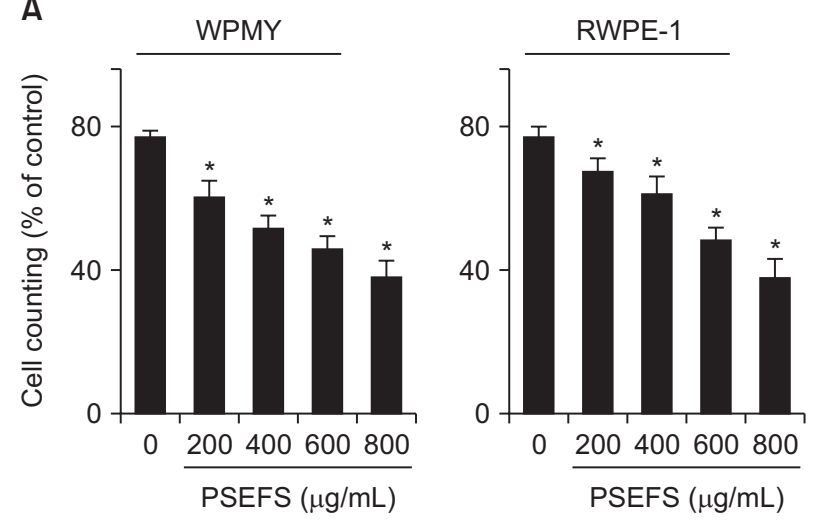

C

Control
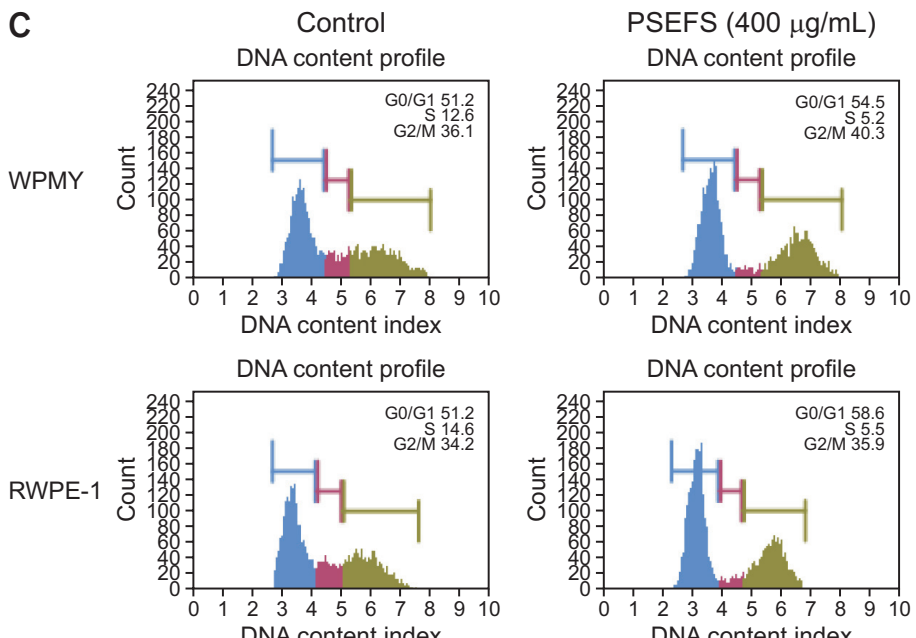

D

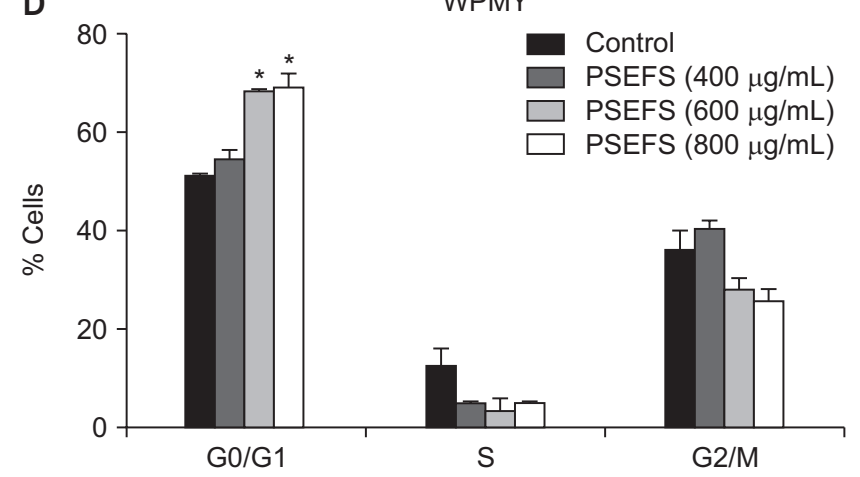

\section{Cell cycle regulation in peanut sprout} extracts cultivated with fermented sawdust medium-treated prostate cells

Following the analysis of cell proliferation, cell cycle distribution was investigated. The flow cytometry analysis of the cell cycle after 24 hours showed that PSEFS strongly induced the accumulation of the
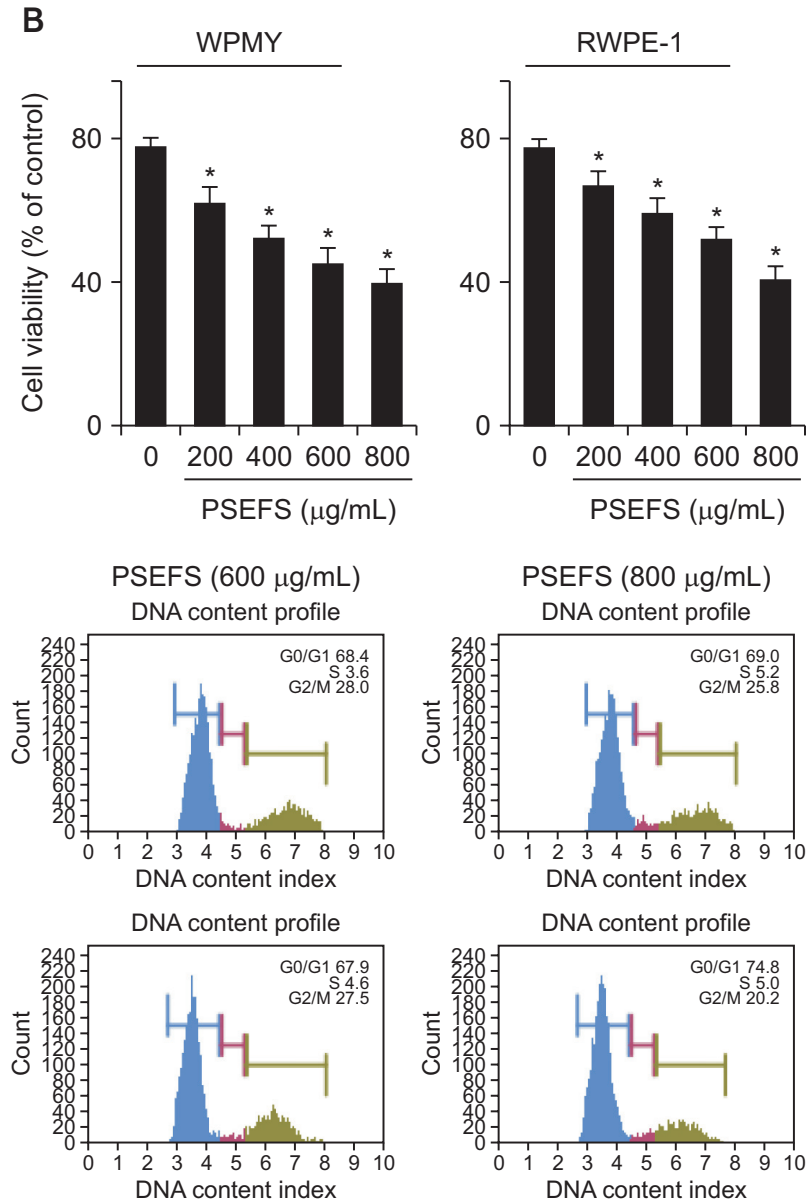

RWPE-1

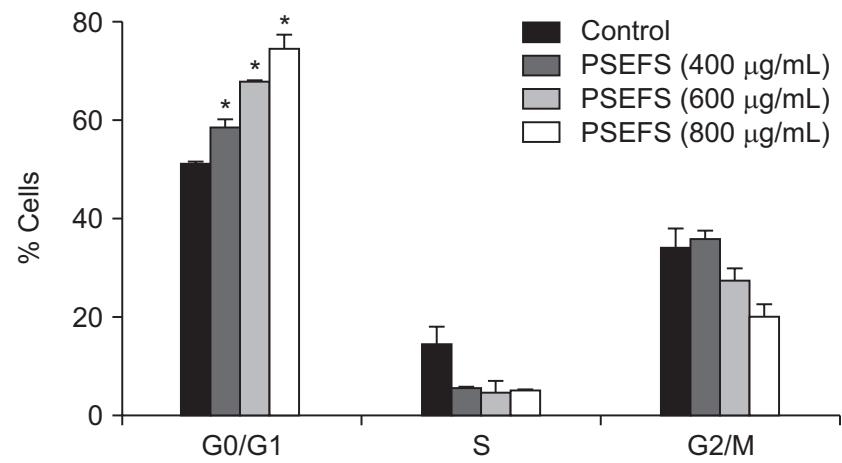

Fig. 2. Peanut sprout extracts cultivated with fermented sawdust medium (PSEFS) suppresses proliferation and induces G1-phase cell cycle arrest in both RWPE-1 and WPMY cells. (A) Both cell lines were treated with various concentrations of PSEFS for 24 hours, followed by cell counting. (B) MTT assay was performed to assess cell viability. (C) Cells are treated with various concentrations of PSEFS for 24 hours, and flow cytometry analysis was performed to evaluate the cell cycle distribution. (D) The percentage of cell population in G1-, S-, and G2/M phases. Values in the bar graph show mean \pm standard deviation of three independent experiments. ${ }^{*} p<0.05$, compared with the control group. 


\section{MEN's HEALTH}

cells in G1-phase of the cell cycle in both cells (Fig. 2C, 2D). In addition, cells at the S- and G2/M-phases were decreased by PSEFS treatment (Fig. 2C, 2D). These results indicated that PSEFS treatment induced an accumulation of the normal human prostate cells in the G1-phase of the cell cycle. To investigate the mechanism of PSEFS-induced G1-phase cell cycle arrest, we examined the expression levels of G1-phase cell cycle regulatory proteins in PSEFS-treated cells. Immunoblot analyses demonstrated that PSEFS treatment decreased the expression levels of cyclin D1 and CDK4 in both prostatic cells (Fig. 3A). However, protein levels of cyclin $\mathrm{E}$ and $\mathrm{CDK} 2$ remained unchanged in both normal prostate cells treated with PSEFS (Fig. 3A). In
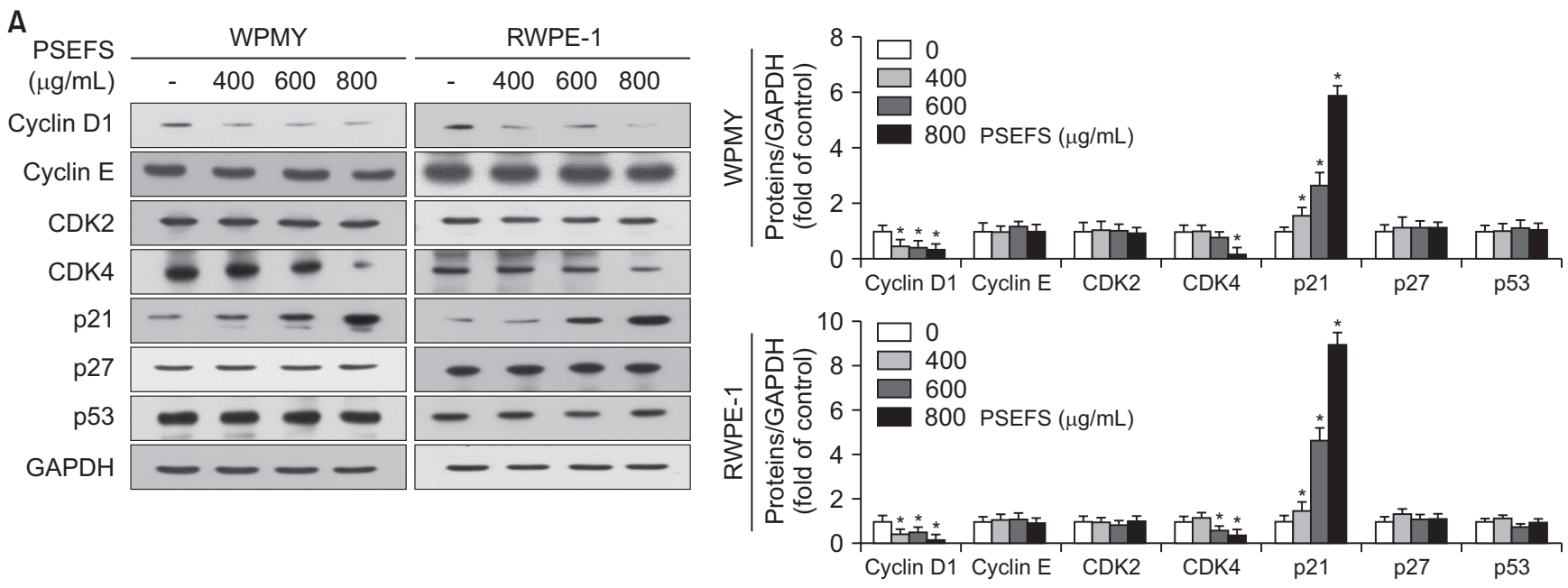

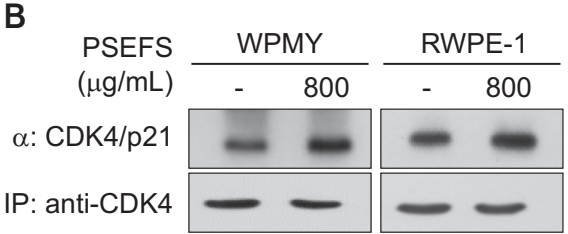

C

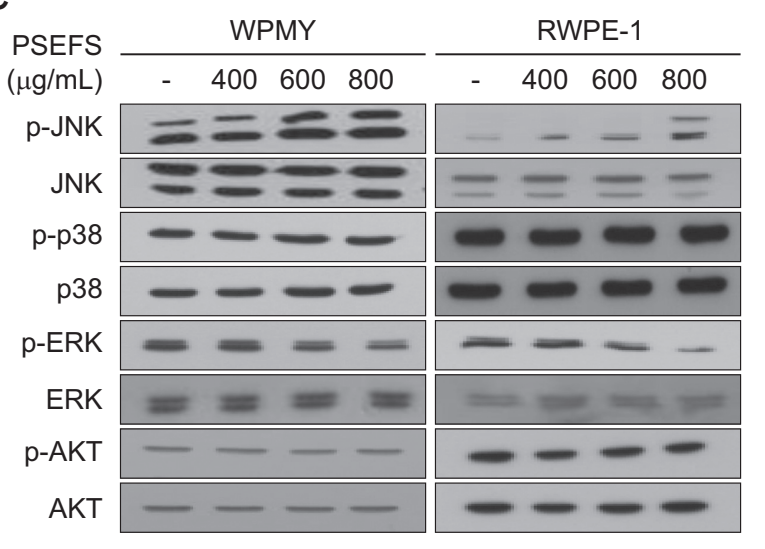

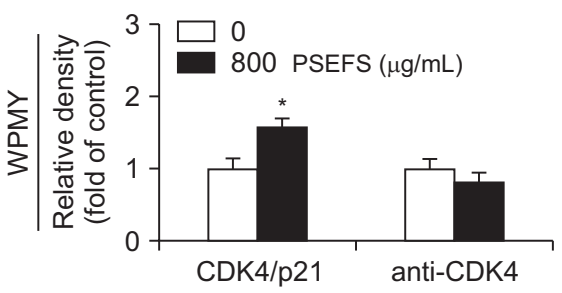

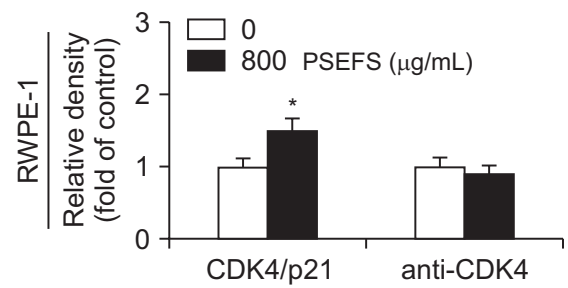

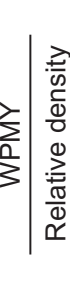
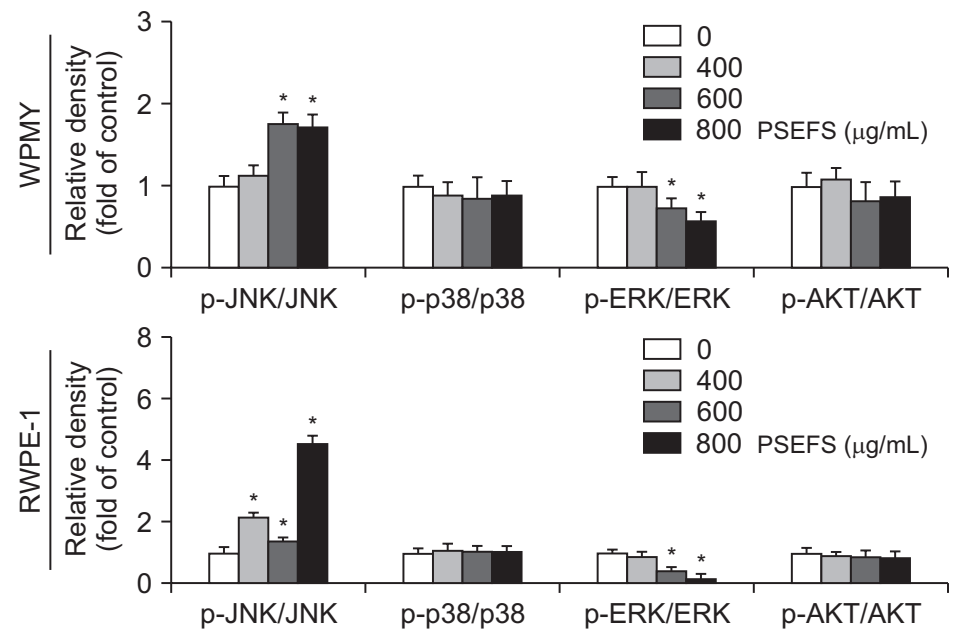

Fig. 3. Effect of peanut sprout extracts cultivated with fermented sawdust medium (PSEFS) on cell-cycle regulators and signaling molecules in prostatic normal cells. Cells were incubated with PSEFS at the indicated concentrations for 24 hours. (A) Changes in regulatory proteins involved in cell-cycle progression were analyzed by immunoblotting. Glyceraldehyde 3-phosphate dehydrogenase (GAPDH) was used as an internal control. (B) Cell lysates were immunoprecipitated (IP) with anti-CDK4 antibody, then immunoblotting was performed with anti-p21WAF1 antibody. (C) Changes in mitogen-activated protein kinases (MAPKs; JNK, p38MAPK, and ERK1/2) and AKT levels were examined by immunoblotting. The level of the phosphorylated form was normalized to the level of the non-phosphorylated form. The bar graphs display fold changes in comparison with controls. Values are shown as the mean \pm standard deviation of three independent experiments. ${ }^{*} p<0.05$, compared with the control group. 
addition, the negative cell cycle regulator p21WAF1 levels were upregulated in PSEFS-treated normal human prostate cells (Fig. 3A). PSEFS treatment did not affect the other negative regulators of the cell cycle, such as p27KIP1 and p53, in both cells (Fig. 3A). It is well known that cell cycle inhibitor p21WAF1 is an important CDK inhibitor [24]. To test the binding affinity between p21WAF1 and CDK4 in PSEFS-treated human normal prostate cells, IP assay was performed using anti-CDK4 antibody followed by immunobinding with anti-p21WAF1 antibody. The IP results showed that PSEFS significantly increased the levels of p21WAF1 bound to CDK4 (Fig. 3B).

\section{Signaling in peanut sprout extracts cultivated with fermented sawdust medium-treated prostate cells}

To determine whether PSEFS-induced inhibition of the proliferation of prostate cells was correlated with changes in the signaling pathways related to cell proliferation, phosphorylation of signaling molecules, including mitogen-activated protein kinases (MAPKs) (ERK, JNK, and p38MAPK) and AKT, was investigated. Immunoblotting indicated that the treatment of RWPE-1 cells with PSEFS inhibited phosphorylation of ERK1/2 but promoted phosphorylation of JNK (Fig. 3C). In contrast, phosphorylation levels of p38MAPK and AKT were not affected by PSEFS (Fig. 3C). Similar results were obtained in WPMY cells (Fig. 3C).
A

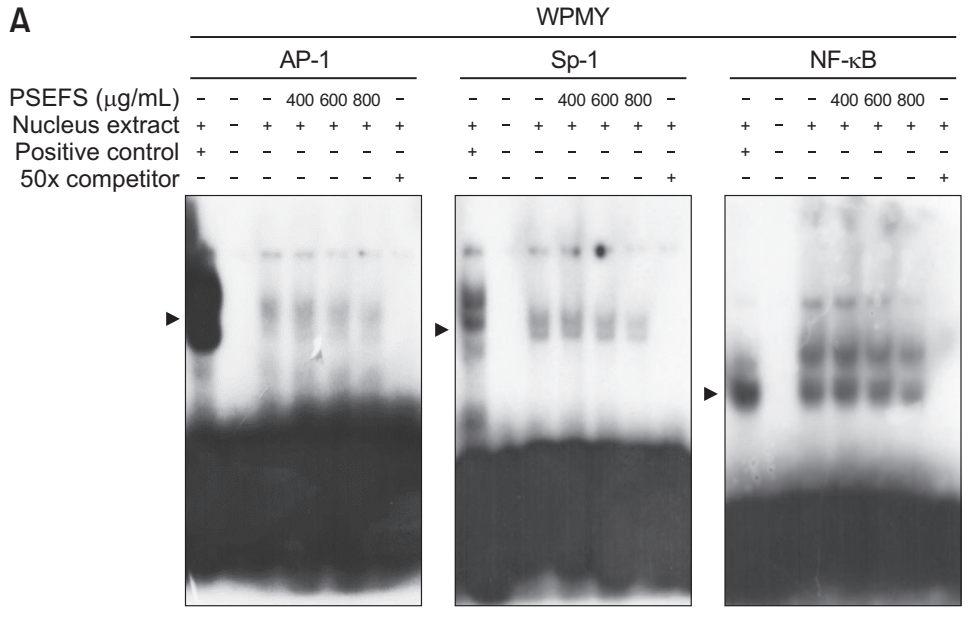

B

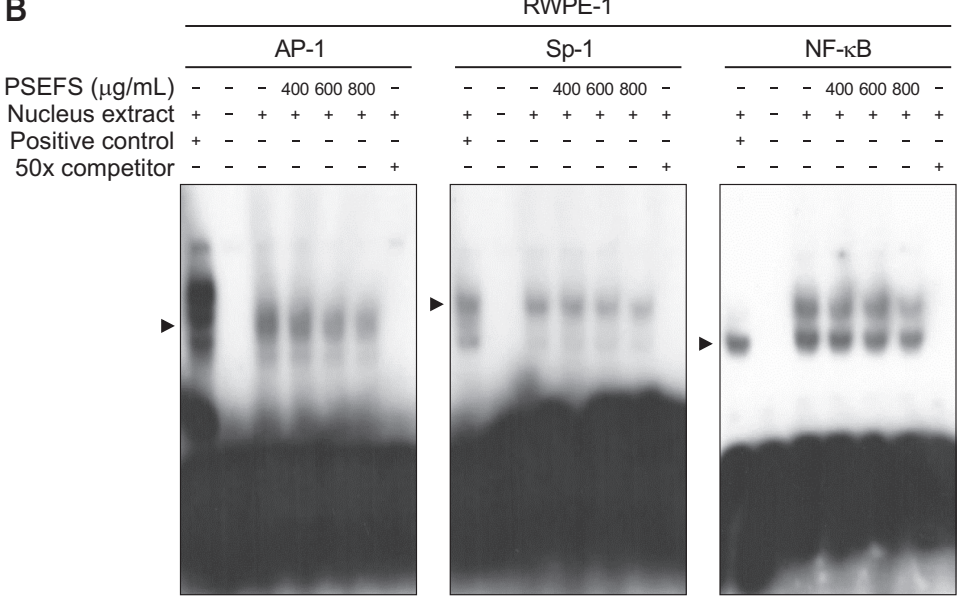

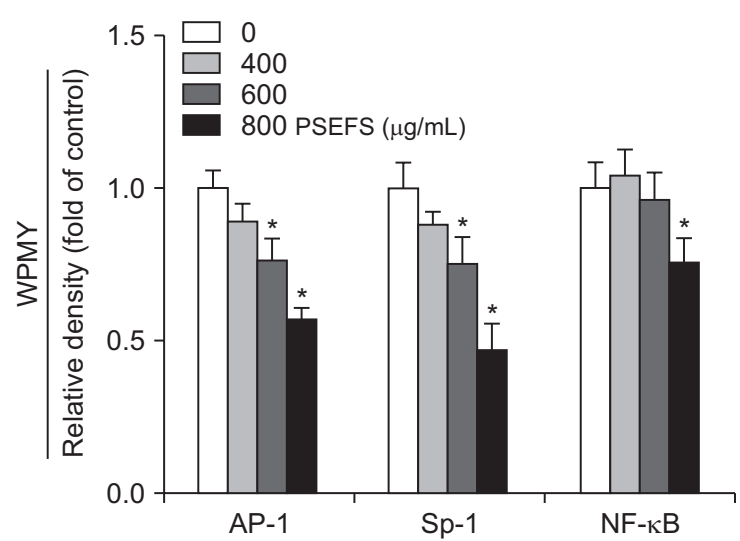

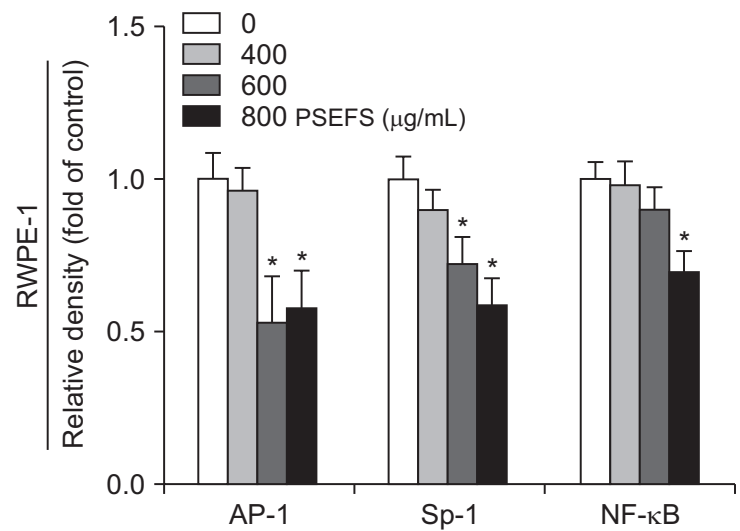

Fig. 4. Peanut sprout extracts cultivated with fermented sawdust medium (PSEFS) suppresses transcriptional binding affinities of the transcription factors nuclear factor- $\kappa$ B (NF- $\kappa$ B), AP-1, and Sp-1 in both types of normal prostatic cells. $(A, B)$ After the preparation of nuclear proteins, electrophoretic mobility shift assay was performed to evaluate transcriptional binding affinities of NF- $\kappa$ B, AP-1, and Sp-1 motifs using radiolabeled oligonucleotide probes. Bar graph represents the fold changes relative to the control. The results are presented as the mean \pm standard deviation from three different experiments. ${ }^{*} p<0.05$, compared to the control. 


\section{Peanut sprout extracts cultivated with} fermented sawdust medium repressed the proliferation of prostate cells via decreased binding activities of the transcription factors nuclear factor- $\kappa \mathrm{B}, \mathrm{Sp}-1$, and AP-1

Previous studies revealed that transcription factors, including nuclear factor-kB (NF-kB), Sp-1, and AP-1, are involved in cell growth and cellular signaling $[11,25]$. To examine the transcriptional effectors by which PSEFS impeded the proliferation of prostate cells, we performed an EMSA assay using the nuclear extracts. As shown in Fig. 4, the EMSA results indicated that PSEFS treatment abrogated the binding affinity of NF- $\mathrm{kB}, \mathrm{Sp}-1$, and AP-1 in both types of prostate cells.

\section{Prostatic hyperplasia markers in peanut sprout extracts cultivated with fermented sawdust medium-treated prostate cells} We next investigated the effects of PSEFS on the expression levels of prostatic proliferation markers (5o-reductase, AR, fibroblast growth factor [FGF], Bcl-2, and Bax) in prostate cells. PSEFS treatment abrogated the expression levels of $5 \alpha$-reductase, AR, and FGF in both RWPE-1 and WPMY cells (Fig. 5). In addition, immunoblotting indicated that anti-proliferative marker Bcl-2 protein levels in both cells was reduced in the presence of PSEFS (Fig. 5). Furthermore, the treatment of both cells with PSEFS increased Bax protein levels (Fig. 5).

\section{Peanut sprout extracts cultivated with} fermented sawdust medium ameliorated prostatic hyperplasia in a testosterone propionate-induced rat model of benign prostatic hyperplasia

An in vivo TP-induced BPH rat model was used to expand the observation of the anti-proliferative effects of PSEFS on prostatic cells in vitro. To compare the inhibitory effects on $\mathrm{BPH}$, finasteride was administered as a positive control. The testosterone (testosterone propionate, TP)-administered rats showed an increase in prostate size compared with the rats in the control group (Con) (Fig. 6A, 6B). Treatment of PSEFS significantly reduced the size of the prostate gland in the TP-induced rat model (Fig. 6A, 6B). More specifically, prostate weight $(\mathrm{PW})$ to body weight $(\mathrm{BW})$ ratio in the testosterone-treated group notably increased compared to that in the Con group (Fig. 6B). In the rats treated with PSEFS, the testosterone-induced PW/BW index was lower (Fig. 6B). Furthermore, the DHT concentrations in the prostate of the TP-induced BPH group were higher than those of the control group (Fig. 6C). The increase of the DHT levels in testosterone-treated rats was reversed by the administration of PSEFS (Fig. $6 C)$. Subsequently, histologic changes were analyzed in the prostate gland using H\&E and Ki-67 stainings. Those results showed that the prostates in testosteronetreated BPH groups showed signs of prostatic hyperplasia, such as multiple layers of epithelial cells and
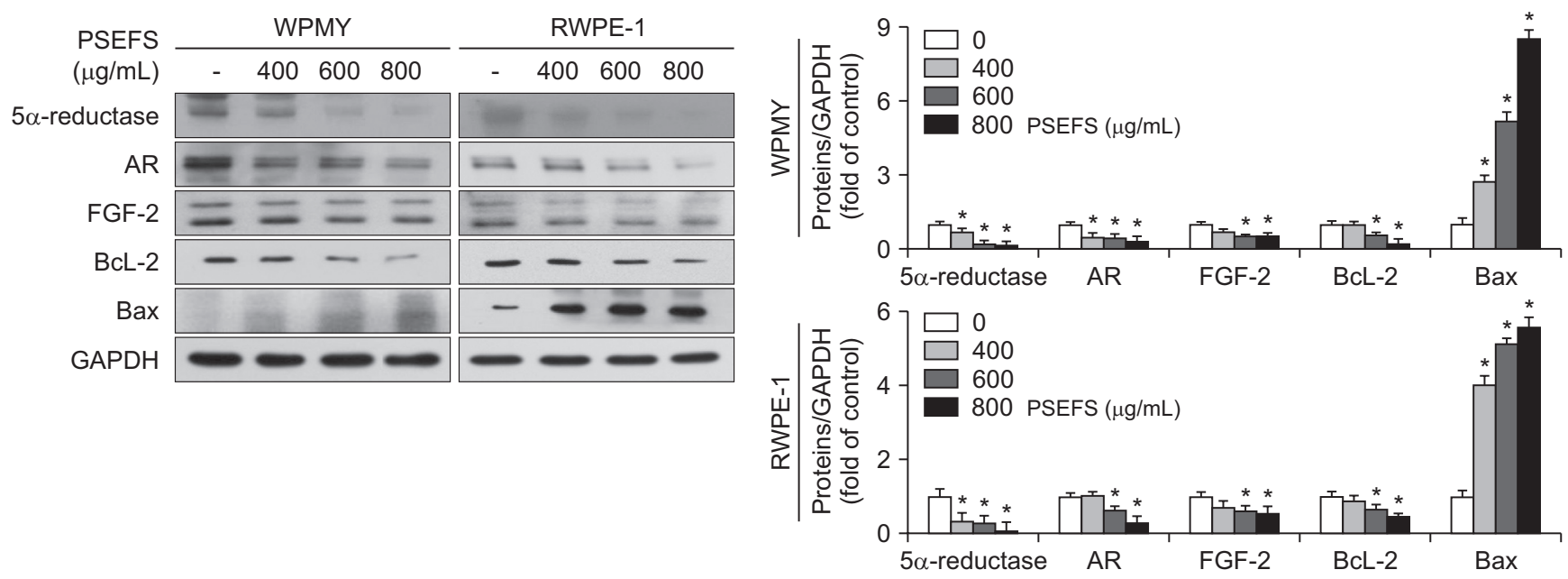

Fig. 5. The expression levels of prostatic proliferation markers in peanut sprout extracts cultivated with fermented sawdust medium (PSEFS)treated prostatic cells. The cells were treated with PSEFS for 24 hours, and then cell lysates were prepared. Expression levels of benign prostatic hyperplasia-associated markers ( $5 \alpha$-reductase, androgen receptor [AR], fibroblast growth factor [FGF], Bcl-2, and Bax) were analyzed by immunoblotting. The bar graphs display fold changes in comparison with the controls. Values are shown as the mean \pm standard deviation of three independent experiments. GAPDH: glyceraldehyde 3-phosphate dehydrogenase. ${ }^{*} p<0.05$, compared with the control group. 
A

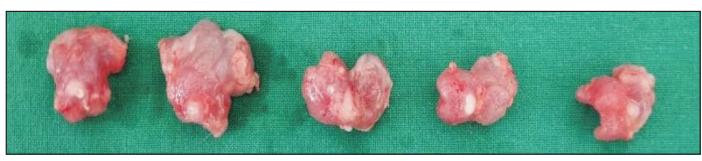

B

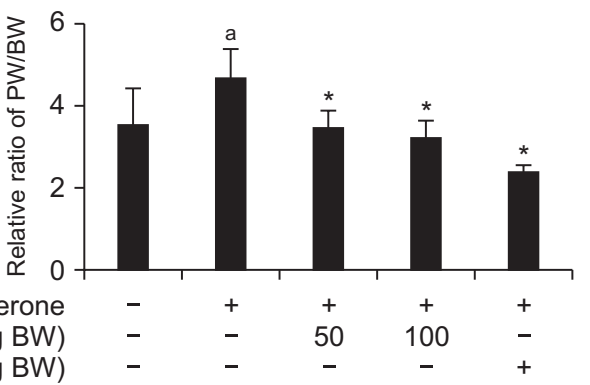

c PSEFS (mg/kg BW) Finasteride $(6 \mathrm{mg} / \mathrm{kg} \mathrm{BW})$

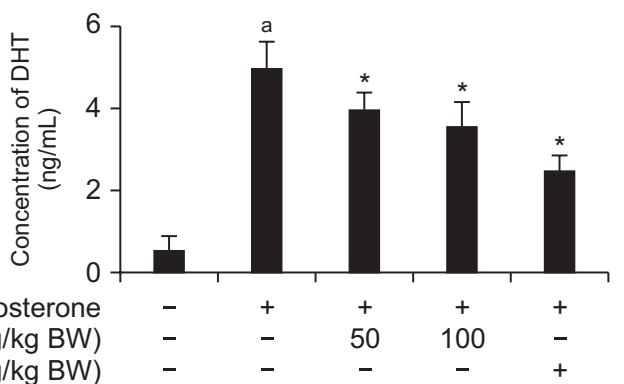

D

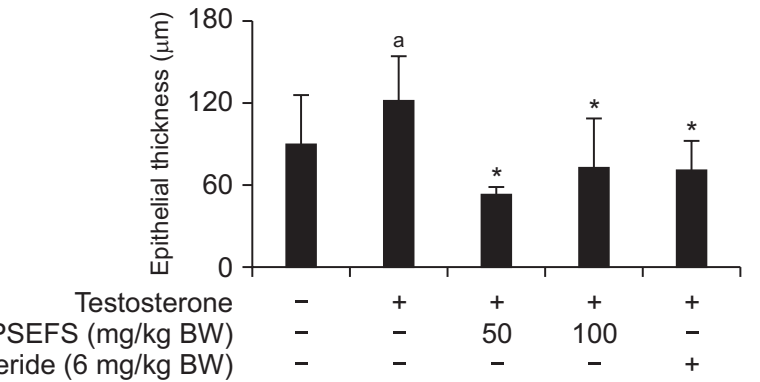
PSEFS $(\mathrm{mg} / \mathrm{kg}$ BW
Finasteride $(6 \mathrm{mg} / \mathrm{kg} \mathrm{BW})$ Finasteride $(6 \mathrm{mg} / \mathrm{kg} \mathrm{BW})$
$E$

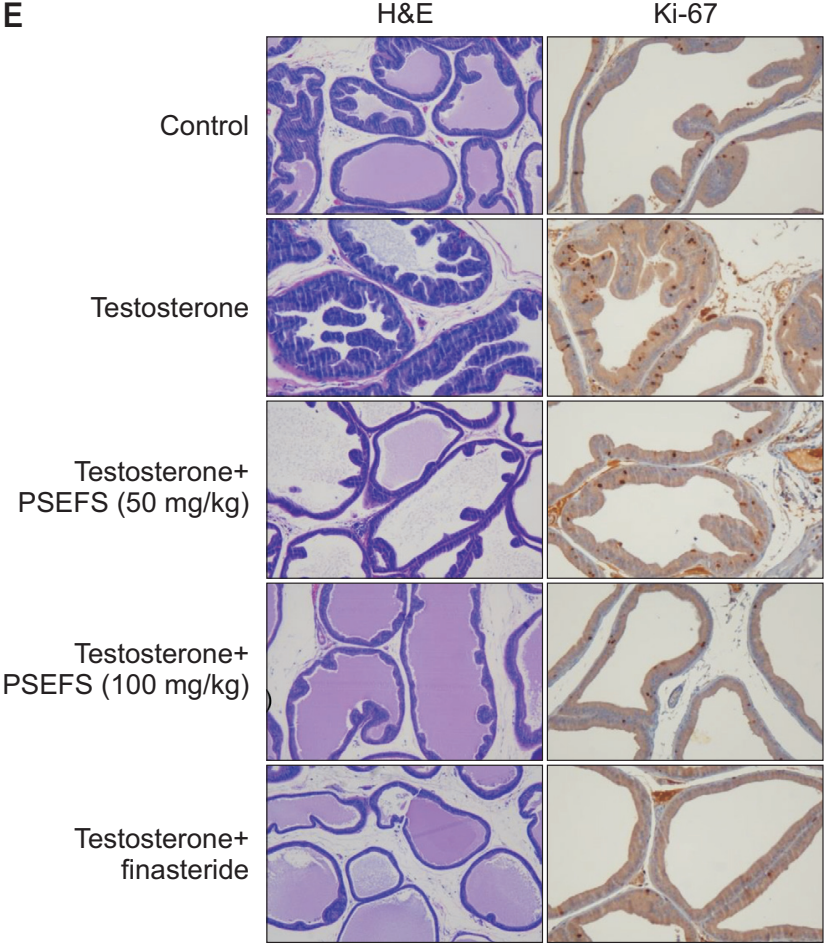

Fig. 6. Effect of peanut sprout extracts cultivated with fermented sawdust medium (PSEFS) on enlarged prostate in a testosterone propionateinduced benign prostatic hyperplasia rat model. (A) Representative images of prostatic tissues from each experimental group were presented. (B) Analysis of prostate weight to body weight (BW) ratio was evaluated. (C) Dihydrotestosterone (DHT) levels were analyzed in prostate tissues using the ELISA kit. (D) The thickness of epithelium tissue from prostate was estimated in prostate tissues. (E) Prostatic tissues were fixed and stained with H\&E and Ki-67 (40x magnification). Values are presented as the mean \pm standard deviation; $\mathrm{n}=7 .{ }^{\mathrm{a}} \mathrm{p}<0.05$, compared to the control group; ${ }^{*} p<0.05$, compared to the testosterone group.

thickening of the muscle layers, in comparison with the con group (Fig. 6E). However, the administration of PSEFS remarkably alleviated TP-induced hyperplastic changes compared with the BPH group (Fig. 6E). Finally, objective assessment in the histological analysis was confirmed by measuring the thickness of epithelium tissue from prostate (TETP). Increased TETP index in the BPH group was reduced by the administration of PSEFS (Fig. 6D).

\section{Peanut sprout extracts cultivated with fermented sawdust medium reduced prostatic hyperplasia via regulation of the prostatic proliferation markers in a testosterone propionate-induced benign prostatic hyperplasia rat model}

The effects of PSEFS in vivo on AR signaling markers (5 $\alpha$-reductase, AR, and FGF) were similar to the in vitro results (Fig. 7). In addition, the regulation of apoptosis-associated molecules (Bcl-2 and Bax) in prostates from PSEFS-treated rats was similar to the in vitro data (Fig. 7). 

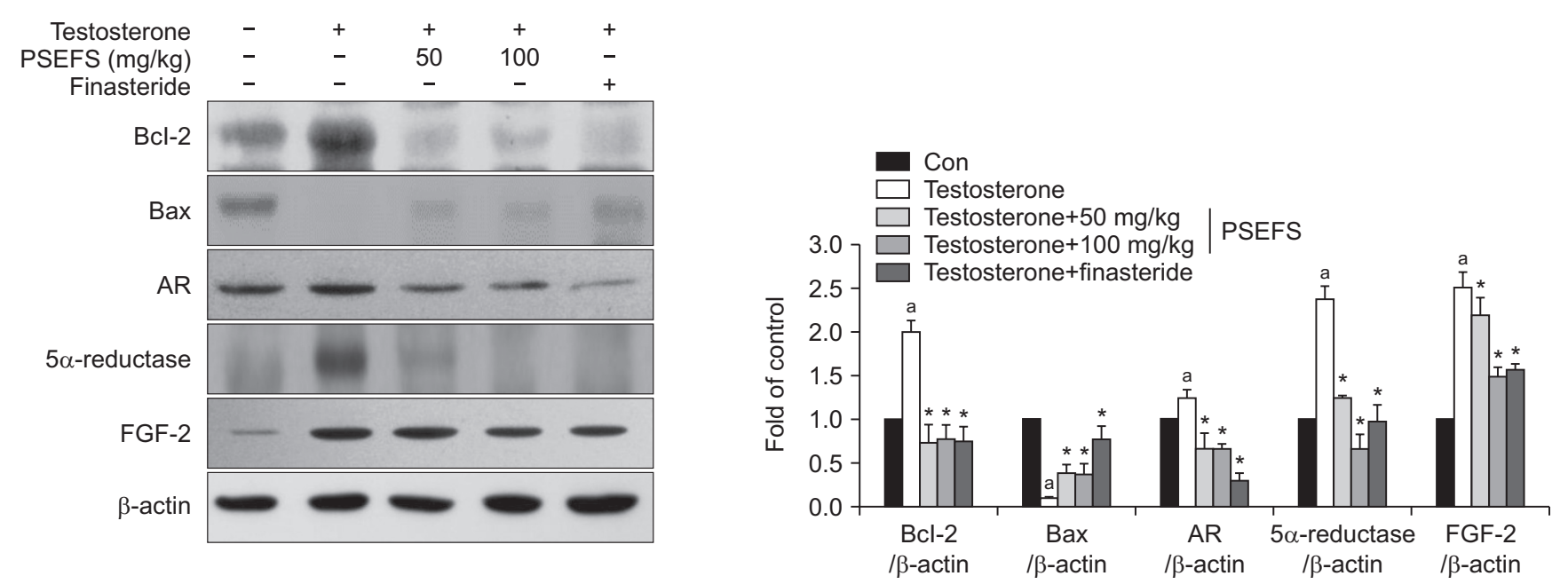

Fig. 7. Effect of peanut sprout extracts cultivated with fermented sawdust medium (PSEFS) on prostatic proliferation markers in the testosterone propionate-induced benign prostatic hyperplasia rat model. The expression level of $5 \alpha$-reductase, androgen receptor (AR), fibroblast growth factor (FGF), Bcl-2, and Bax in prostatic tissues was examined by immunoblotting. Bar graphs represent the fold changes in expression levels compared with the control (Con). Values are presented as the mean \pm standard deviation; $\mathrm{n}=7 .{ }^{\mathrm{a}} \mathrm{p}<0.05$, compared to the Con group; ${ }^{*} \mathrm{p}<0.05$, compared to the testosterone group.

\section{DISCUSSION}

It has been demonstrated that RES has many benefits such as anti-cardiovascular effect, anti-tumor effect, anti-oxidant effect, and anti-BPH effect [13,16,26]. Since previous studies have suggested that the peanut sprouts contain high amounts of RES, we investigated the content of RES in 5 types of the Korean peanut varieties (Sindae, Inhua-C, Inhua-W, Inhua-L, and Yesan) germinated for 9 days using sawdust medium fermented for 45 days. The highest levels of RES were found in the Yesan variety that was used in the subsequent experiments. Comparing the commercial peanut sprouts cultivated with HP, peanut sprout (Yesan) cultivated by fermented sawdust medium revealed the higher level of RES content. Therefore, the efficacy of peanut sprout extracts (Yesan) cultivated with fermented sawdust medium against BPH was subsequently tested in vitro and in vivo.

The crucial pathological hallmark is the abnormal proliferation of prostate cells caused by an imbalance between cell growth and cell death $[2,7,8]$. PSEFS treatment inhibited the proliferation of both human normal epithelial cells and stromal cells. The cell cycle progression is considered to play a central role in cellular responses including cell proliferation [24,27]. Cell cycle is tightly regulated by G1-, S-, and G2/M-phase cell cycle transition checkpoints [24,27]. Treatment of the prostate cells with PSEFS resulted in the G1- phase cell cycle arrest. CDK4/cyclin D1 and CDK2/ cyclin E complexes are key regulators in the G1-phase cell cycle progression in eukaryotic cells [27]. We next investigated the level of CDKs and cyclins in PSEFStreated prostate cells. PSEFS treatment repressed the protein levels of CDK4 and cyclin D1 without change of CDK2 and cyclin E. In addition, the G1-phase cell cycle was controlled by negative regulators p21WAF1 and p27KIP1 and tumor suppressor molecule p53 [24,27]. Treatment of PSEFS induced p21WAF1 level in both prostate cells without alteration of p27Kip1 and p53. These results demonstrate that PSEFS inhibited the proliferation of prostate cells via p21WAF1-mediated G1-phase cell cycle arrest by decreased level of CDK4/ cyclin D1.

MAPKs and AKT are the main signaling molecules that are involved in the proliferation, cellular retardation, and cell cycle regulation [7,8,28,29]. A previous report has demonstrated that ERK1/2 phosphorylation was a key signaling factor in BPH model [10]. However, the precise role of JNK signaling in $\mathrm{BPH}$ is yet to be elucidated. In the present study, PSEFS treatment decreased the phosphorylation of ERK1/2, whereas the phosphorylation of JNK was increased in both types of prostate cells. Both p38MAPK and AKT phosphorylation were not altered in the presence of PSEFS. These results indicate that the increased ERK1/2 signaling and reduced JNK signaling pathway is involved in PSEFS-induced inhibition of proliferation of prostate 
cells. In addition, transcription factors NF- $\mathrm{B}$, Sp-1, and AP-1 levels were also downregulated in PSEFS-treated prostate cells. Indeed, transcription factors regulate the expression of multiple genes involved in cell proliferation, cellular stress, immune responses, and pathological conditions [7-9,11]. Previous studies have shown that NF- $\mathrm{B}$, E2F-1, and AP-1 maintain the proliferation of human prostatic cells $[9,11,30]$. To date, no information was found on the relationship between Sp-1 and prostate cells. Our data suggest that transcription factors are important regulators responsible for the PSEFSinduced inhibition of proliferation of human prostate cells. Further studies are needed to clarify the role of transcription factors, especially $\mathrm{Sp}-1$, in $\mathrm{BPH}$ pathogenesis.

We next quantified the levels of molecular markers responsible for the suppressive effects of PSEFS on BPH development and progression. Although the mechanisms of $\mathrm{BPH}$ development is not fully understood, it is well established that prostate enlargement is involved and modulated at the level of 5AR-AR axis, DHT, FGF, Bcl-2, and Bax [3,7,8]. In the present study, the levels of 5AR, AR, FGF, and Bcl-2 were downregulated, whereas Bax level was upregulated in PSEFStreated prostate cells in vitro. In addition, TP-induced $\mathrm{BPH}$ rat model was used to investigate the effects of PSEFS on the underlying pathologic growth and hormonal disturbance of prostate tissue in vivo. Administration of PSEFS (100 mg/kg) for 4 weeks significantly decreased both the size and the weight of prostate tissues with no signs of death compared with those of the TP-induced BPH groups. Furthermore, comparing prostate tissues in TP-induced BPH rat group, the thickness of prostate tissues also reduced, after PSEFS. The effect of PSEFS on TP-induced prostatic cell proliferation in the BPH rat model was verified by H\&E and Ki-67 immunostaining. Finally, the levels of molecular makers (5AR-AR axis, FGF, Bcl-2, and Bax) responsible for the TP-induced pathologic growth of prostate tissues were confirmed in PSEFS-treated BPH rat model. Interestingly, the in vivo study demonstrated that the low levels of DHT were observed by PSEFS administration in comparison with the TP-induced $\mathrm{BPH}$ rat model. These results from the present study demonstrated that PSEFS might reduce the DHT level via inhibition of the 5AR-AR axis, leading to the blockade of BPH enlargement. Collectively, our results indicate that PSEFS exerts the suppressive effects against BPH via regulation of the 5AR-AR-DHT axis, growth factor, and apoptosis-related molecules.

\section{CONCLUSIONS}

Taken together, the inhibitory effects of PSEFS in both WPMY and RWPE-1 cells were strongly correlated with decreased cell proliferation, p21-mediated G1-phase cell cycle arrest, altered signaling pathway, dysregulated transcription factors, and reduced level of BPH-associated molecular markers. Animal studies confirmed the suppression of pathological prostatic features against the TP-induced rat BPH model of PSEFS with no signs of death. Our results show the beneficial effects of PSEFS against BPH. Thus, PSEFS can be developed into a preventive or a therapeutic reagent in $\mathrm{BPH}$ for the BPH patents through its anti-hyperplasia effects.

\section{ACKNOWLEDGEMENTS}

This work was supported by Korea Institute of Planning and Evaluation for Technology in Food, Agriculture and Forestry (IPET) through Technology Commercialization Support Program, funded by Ministry of Agriculture, Food and Rural Affairs (MAFRA) (8170213). This research was also supported by Basic Science Research Program through the National Research Foundation of Korea (NRF) funded by the Ministry of Education (NRF-2018R1A6A1A03025159).

\section{Conflict of Interest}

The authors have nothing to disclose.

\section{Author Contribution}

Conceptualization: BWK, JHS, SCM, SKM, WRK. Formal analysis: BH, HJC, JHS. Funding acquisition: BWK, WRK. Investigation: $\mathrm{BH}, \mathrm{BKM}$, HJC. Methodology: BH, BKM, HJC. Project administration: SCM, SKM. Supervision: JWK, KK, WJK. Validation: JWK, KK, WJK. Writing - original draft: JHS, SCM, SKM. Writing - review \& editing: JHS, SKM.

\section{Supplementary Materials}

Supplementary materials can be found via https://doi. org/10.5534/wjmh.190173. 


\section{MEN's HEALTH}

\section{Data Sharing Statement}

The data required to reproduce these findings cannot be shared at this time as the data also forms part of an ongoing study.

\section{REFERENCES}

1. Berry SJ, Coffey DS, Walsh PC, Ewing LL. The development of human benign prostatic hyperplasia with age. J Urol 1984;132:474-9.

2. O'Leary MP. Lower urinary tract symptoms/benign prostatic hyperplasia: maintaining symptom control and reducing complications. Urology 2003;62(3 Suppl 1):15-23.

3. McConnell JD. Prostatic growth: new insights into hormonal regulation. Br J Urol 1995;76 Suppl 1:5-10.

4. Pirozzi L, Sountoulides P, Castellan P, Presicce F, Lombardo R, Romero M, et al. Current pharmacological treatment for male LUTS due to BPH: dutasteride or finasteride? Curr Drug Targets 2015;16:1165-71.

5. Corona G, Tirabassi G, Santi D, Maseroli E, Gacci M, Dicuio $\mathrm{M}$, et al. Sexual dysfunction in subjects treated with inhibitors of $5 \alpha$-reductase for benign prostatic hyperplasia: a comprehensive review and meta-analysis. Andrology 2017;5:671-8.

6. Nickel JC, Gilling P, Tammela TL, Morrill B, Wilson TH, Rittmaster RS. Comparison of dutasteride and finasteride for treating benign prostatic hyperplasia: the Enlarged Prostate International Comparator Study (EPICS). BJU Int 2011;108:388-94.

7. Izumi K, Mizokami A, Lin WJ, Lai KP, Chang C. Androgen receptor roles in the development of benign prostate hyperplasia. Am J Pathol 2013;182:1942-9.

8. Lamont KR, Tindall DJ. Minireview: alternative activation pathways for the androgen receptor in prostate cancer. Mol Endocrinol 2011;25:897-907.

9. Jin BR, Kim HJ, Kim EY, Chung TW, Ha KT, An HJ. 6'-sialyllactose ameliorates in vivo and in vitro benign prostatic hyperplasia by regulating the E2F1/pRb-AR pathway. Nutrients 2019;11:E2203.

10. Youn DH, Park J, Kim HL, Jung Y, Kang J, Lim S, et al. Berberine improves benign prostatic hyperplasia via suppression of 5 alpha reductase and extracellular signal-regulated kinase in vivo and in vitro. Front Pharmacol 2018;9:773.

11. Austin DC, Strand DW, Love HL, Franco OE, Jang A, Grabowska MM, et al. NF- $\kappa \mathrm{B}$ and androgen receptor variant expression correlate with human $\mathrm{BPH}$ progression. Prostate 2016;76:491-511.

12. de Vries K, Strydom M, Steenkamp V. Bioavailability of resveratrol: possibilities for enhancement. J Herb Med 2018;11:71-
Jun-Hui Song, et al: Effect of Peanut Sprout Extracts in BPH

7.

13. de la Lastra CA, Villegas I. Resveratrol as an antioxidant and pro-oxidant agent: mechanisms and clinical implications. Biochem Soc Trans 2007;35(Pt 5):1156-60.

14. Howitz KT, Bitterman KJ, Cohen HY, Lamming DW, Lavu S, Wood JG, et al. Small molecule activators of sirtuins extend Saccharomyces cerevisiae lifespan. Nature 2003;425:191-6.

15. Kang H, Kim J, Kwon S, Park K, Kang J, Seo K. Antioxidative effects of peanut sprout extracts. J Korean Soc Food Sci Nutr 2010;39:941-6

16. Li C, Hu WL, Lu MX, Xiao GF. Resveratrol induces apoptosis of benign prostatic hyperplasia epithelial cell line (BPH-1) through p38 MAPK-FOXO3a pathway. BMC Complement Altern Med 2019;19:233.

17. Ge M, Chen G, Hong J, Huang X, Zhang L, Wang L, et al. Screening for formulas of complex substrates for seedling cultivation of tomato and marrow squash. Proced Environ Sci 2012;16:606-15.

18. El-Kazzaz KA, El-Kazzaz AA. Soilless agriculture a new and advanced method for agriculture development: an introduction. Agri Res Tech 2017;3:63-72.

19. Meng X, Dai J, Zhang Y, Wang X, Zhu W, Yuan X, et al. Composted biogas residue and spent mushroom substrate as a growth medium for tomato and pepper seedlings. J Environ Manage 2018;216:62-9.

20. Onwosi CO, Igbokwe VC, Odimba JN, Eke IE, Nwankwoala MO, Iroh IN, et al. Composting technology in waste stabilization: on the methods, challenges and future prospects. J Environ Manage 2017;190:140-57.

21. Choi JY, Choi DI, Lee JB, Yun SJ, Lee DH, Eun JB, et al. Ethanol extract of peanut sprout induces Nrf2 activation and expression of antioxidant and detoxifying enzymes in human dermal fibroblasts: implication for its protection against UVB-irradiated oxidative stress. Photochem Photobiol 2013;89:453-60.

22. Limmongkon A, Nopprang P, Chaikeandee P, Somboon T, Wongshaya P, Pilaisangsuree V. LC-MS/MS profiles and interrelationships between the anti-inflammatory activity, total phenolic content and antioxidant potential of Kalasin 2 cultivar peanut sprout crude extract. Food Chem 2018;239:56978.

23. Kang NE, Ha AW, Woo HW, Kim WK. Peanut sprouts extract (Arachis hypogaea L.) has anti-obesity effects by controlling the protein expressions of PPAR $\gamma$ and adiponectin of adipose tissue in rats fed high-fat diet. Nutr Res Pract 2014;8:158-64.

24. Harper JW, Adami GR, Wei N, Keyomarsi K, Elledge SJ. The p21 Cdk-interacting protein Cip1 is a potent inhibitor of G1 cyclin-dependent kinases. Cell 1993;75:805-16. 
25. Lee SJ, Cho SC, Lee EJ, Kim S, Lee SB, Lim JH, et al. Interleukin-20 promotes migration of bladder cancer cells through extracellular signal-regulated kinase (ERK)-mediated MMP-9 protein expression leading to nuclear factor $(\mathrm{NF}-\kappa \mathrm{B})$ activation by inducing the up-regulation of $\mathrm{p} 21$ (WAF1) protein expression. J Biol Chem 2013;288:5539-52.

26. Ramírez-Garza SL, Laveriano-Santos EP, Marhuenda-Muñoz M, Storniolo CE, Tresserra-Rimbau A, Vallverdú-Queralt A, et al. Health effects of resveratrol: results from human intervention trials. Nutrients 2018;10:E1892.

27. Sherr CJ. Cancer cell cycles. Science 1996;274:1672-7.

28. Gerhardt D, Bertola G, Dietrich F, Figueiró F, Zanotto-Filho A, Moreira Fonseca JC, et al. Boldine induces cell cycle arrest and apoptosis in T24 human bladder cancer cell line via regulation of ERK, AKT, and GSK-3 $\beta$. Urol Oncol 2014;32:36.e19.

29. Zheng X, Ou Y, Shu M, Wang Y, Zhou Y, Su X, et al. Cholera toxin, a typical protein kinase A activator, induces G1 phase growth arrest in human bladder transitional cell carcinoma cells via inhibiting the c-Raf/MEK/ERK signaling pathway. Mol Med Rep 2014;9:1773-9.

30. Lin-Tsai O, Clark PE, Miller NL, Fowke JH, Hameed O, Hayward SW, et al. Surgical intervention for symptomatic benign prostatic hyperplasia is correlated with expression of the AP-1 transcription factor network. Prostate 2014;74:669-79. 